\title{
Consequências e agravantes de saúde evidenciados pela COVID-19 em populações indígenas brasileiras: uma revisão integrativa
}

\author{
Health consequences and aggravations evidenced by COVID-19 in Brazilian indigenous \\ populations: an integrative review
}

Consecuencias y agravamientos en la salud evidenciados por COVID-19 en poblaciones indígenas brasileñas: una revisión integradora

Jefter Haad Ruiz da Silva ORCID: https://orcid.org/0000-0002-1322-640X Universidade Federal do Amazonas, Brasil E-mail: jefterhaad@ hotmail.com Francisco Ferreira Barcelar Junior ORCID: https://orcid.org/0000-0002-8481-1083 Centro Universitário do Norte, Brasil E-mail: barcellarjunior@gmail.com

Jéssica Lourdes de Aguiar Gonçalves ORCID: https://orcid.org/0000-0002-4351-1922 Universidade do Estado do Amazonas, Brasil E-mail: jessica_lurdes@hotmail.com Râmyla Leitão Resk ORCID: https://orcid.org/0000-0002-2931-3057 Universidade do Estado do Amazonas, Brasil E-mail: ramyllareesk@ hotmail.com

Maria Alice Barbosa da Silva ORCID: https://orcid.org/0000-0003-0876-7356 Universidade Federal de Pernambuco, Brasil E-mail: marialicebarbosadasilva@gmail.com

Yasmin Michelle Tupinambá da Silva ORCID: https://orcid.org/0000-0002-9488-9896 Universidade do Estado do Amazonas, Brasil

E-mail: yasmintupinamba96@gmail.com

Elise Costa dos Santos

ORCID: https://orcid.org/0000-0003-1345-2287 Escola Superior Batista do Amazonas, Brasil

E-mail: elisepsic@outlook.com

Juliana Matos Chaves

ORCID: https://orcid.org/0000-0002-9920-186X Universidade do Estado do Amazonas, Brasil E-mail: jumachajumacha@hotmail.com

Mylla Cristie Campelo Monteiro ORCID: https://orcid.org/0000-0001-7543-1030 Universidade do Estado do Amazonas, Brasil E-mail: allymonteiro@ outlook.com

Samuel Filipe Santos de Oliveira ORCID: https://orcid.org/0000-0003-3708-3682 Universidade Venda Nova do Imigrante, Brasil E-mail: slipe0055@gmail.com

Jéssica Karoline Brasil da Silva Santim ORCID: https://orcid.org/0000-0002-6725-190X Universidade do Estado do Amazonas, Brasil E-mail: jesskaroline3008@gmail.com

\section{Resumo}

Este estudo objetivou realizar uma revisão integrativa acerca dos estudos que identificaram a influência da pandemia na saúde de indígenas brasileiros, a fim de agrupar estas informações e gerar dados pertinentes à uma análise qualitativa. Foram realizadas buscas nas bases de dados Scielo, Pubmed e BVS, utilizando-se os descritores "covid19", "indigenous" e "Brazil" conectados pelo operador booleano "AND". Foram considerados somente artigos científicos publicados nos últimos 05 anos, de acesso gratuito, e cujo conteúdo fosse escrito em língua portuguesa ou inglesa. No total foram encontrados 22 artigos, sendo que 10 deles foram considerados na composição dos resultados. 
Observou-se que os principais fatores da realidade indígena evidenciados pelo contexto pandêmico estão associados às consequências oriundas do isolamento social (falha no suprimento de alimentos, ameaças de grileiros e migração de centros urbanos para aldeias), a barreiras geográficas (dificuldade de atendimento médico devido as rigorosas vigilâncias em fronteiras), e a aspectos culturais (recusa na utilização de medicamentos sintéticos, execução de cerimônias ritualísticas em grupo e confinamento de muitas pessoas em um mesmo ambiente). As etnias indígenas têm se deparado com diversos agravantes ocasionados pela pandemia, o que tem servido de obstáculos à concretização de uma assistência em saúde que faça jus às especificidades deste povo, sendo necessário - frente os desafios impostos pela pandemia - fortalecer setores de atenção específicos, além de garantir a participação de lideranças indígenas no processo de construção de políticas públicas saudáveis.

Palavras-chave: COVID-19; Populações indígenas; Saúde das minorias étnicas; Brasil.

\begin{abstract}
This study aimed to carry out an integrative review of studies that identified the influence of the pandemic on the health of Brazilian indigenous peoples, in order to group this information and generate data relevant to a qualitative analysis. Searches were performed in the Scielo, Pubmed and BVS databases, using the descriptors "covid-19", "indigenous" and "Brazil" connected by the Boolean operator "AND". Only scientific articles published in the last 05 years, with free access, and whose content was written in Portuguese or English, were considered. In total, 22 articles were found, and 10 of them were considered in the composition of the results. It was observed that the main factors of the indigenous reality evidenced by the pandemic context are associated with the consequences arising from social isolation (failure in the supply of food, threats from land grabbers, and migration from urban centers to villages), geographic barriers (difficulty in medical care due to strict border surveillance), and cultural aspects (refusal in the use of synthetic medicines, performance of ritualistic group ceremonies, and confinement of many people in the same environment). Indigenous ethnic groups have faced several aggravations caused by the pandemic, which has served as obstacles to the implementation of health care that does justice to the specificities of this people, being necessary - in view of the challenges posed by the pandemic - to strengthen specific care sectors, in addition to ensuring the participation of indigenous leaders in the process of building healthy public policies.
\end{abstract}

Keywords: COVID-19; Indigenous population; Health of ethnic minorities; Brazil.

\title{
Resumen
}

Este estudio tuvo como objetivo realizar una revisión integradora de estudios que identificaron la influencia de la pandemia en la salud de los pueblos indígenas brasileños, con el fin de agrupar esta información y generar datos relevantes para un análisis cualitativo. Las búsquedas se realizaron en las bases de datos Scielo, Pubmed y BVS, utilizando los descriptores "covid-19", "indígena" y "Brasil” conectados por el operador booleano "AND”. Solo se consideraron artículos científicos publicados en los últimos 05 años, de libre acceso, y cuyo contenido fue escrito en portugués o inglés. En total, se encontraron 22 artículos, y 10 de ellos fueron considerados en la composición de los resultados. Se observó que los principales factores de la realidad indígena evidenciada por el contexto pandémico están asociados a las consecuencias derivadas del aislamiento social (fallas en el suministro de alimentos, amenazas de acaparadores de tierras y migración de los centros urbanos a las aldeas), barreras geográficas (dificultad en la atención médica por estricta vigilancia fronteriza), y aspectos culturales (rechazo en el uso de medicinas sintéticas, realización de ceremonias rituales en grupos y confinamiento de muchas personas en un mismo ambiente). Las etnias indígenas han enfrentado diversos agravios provocados por la pandemia, la cual ha servido como obstáculos para la implementación de una atención de salud que haga justicia a las especificidades de este pueblo, siendo necesario ante los desafíos que plantea la pandemia - fortalecer la atención específica. sectores, además de asegurar la participación de los líderes indígenas en el proceso de construcción de políticas públicas saludables.

Palabras clave: COVID-19; Poblaciones indigenas; Salud de las minorías étnicas; Brasil.

\section{Introdução}

A doença infecciosa COVID-19, causada pelo vírus SARS-CoV-19, surtiu efeitos devastadores à população mundial quando, no segundo semestre de 2019, iniciou uma torrente de contaminações que culminou em mais de 2,9 milhões de mortes na população mundial. Tem como principais sinais e sintomas a febre, tosse seca, mialgia, dispneia e linfopenia, sendo as secreções advindas do sistema respiratório o seu principal meio de propagação. Indivíduos com idade acima de 60 anos, assim como pessoas com comorbidades preexistentes (tais como obesidade, doença renal crônica, diabetes, etc.) são as mais propensas ao agravamento de infecções oriundas do vírus - sendo tais evidências as mais disseminadas no meio científico atualmente (Belasco \& Fonseca, 2020; Cascacella et al., 2021).

Frente às evidências de seu impacto social, a pandemia influenciou tanto aspectos culturais quanto de convivência entre comunidades, uma vez que diversas incertezas moldaram a tomada de decisões governamentais em momentos críticos, 
culminando na incisiva tomada de decisões emergenciais, que, por vezes, foram consideradas impopulares, mas de fundamental importância à preservação do bem-estar público. Quanto as múltiplas consequências acentuadas pelo contexto pandêmico, é possível citar: a diminuição da renda familiar, o aumento da taxa de desemprego, a piora no estado de saúde geral, bem como o aumento de casos de depressão, ansiedade e nervosismo (Almeida et al., 2021). Contudo, tais implicações não estão restritas apenas à moradores de grandes centros urbanos, estendendo-se, também, aos povos interioranos e aos subgrupos historicamente vulneráveis, como - por exemplo - populações indígenas de terras brasileiras.

A $1^{\text {a }}$ Conferência Nacional de Proteção à Saúde Indígena, em 1986, expôs as especificidades dos povos indígenas a partir do ponto de vista das lideranças participantes na garantia de direitos salutares a esta população, sendo este um marco importante à consolidação de possíveis estratégias capazes de incluir a integralidade do cuidado na realidade das aldeias (Mendes et al., 2018). A incorporação da assistência médica a partir do Sistema Único de Saúde (SUS), deu-se através da criação do Subsistema de Atenção à Saúde Indígena (SASI) (Lei 9.836/1999) que atua considerando as inerentes especificidades desta população - principalmente no que se refere às características culturais, sociais e epidemiológicas deste grupo. A partir de 2010, a secretaria de gestão, sob o amparo do Ministério da Saúde, passou a ser constituída pela Secretaria Especial de Saúde Indígena (SESAI), cuja descentralização dispõe-se através da criação de Distritos Sanitários Especiais Indígenas (DSEI) que compõem sua assistência através da constituição de Polo Base, Casas de Saúde Indígena (CASAIs), e equipes multidisciplinares. A disposição geográfica destes distritos transpõe os limites dos estados brasileiros, e possui instâncias de participação popular em conjunto à gestão local - propiciando a formação de conselhos essenciais ao planejamento, atuação e monitoração das atividades em saúde (Scalco et al., 2020). O Ministério da Saúde gerencia este subsistema a partir dos 225 povos indígenas alocados no Brasil, onde a SESAI é responsável por garantir a proteção, a educação em saúde, a promoção e a recuperação destas comunidades (Cardoso, 2015).

A saúde dos povos indígenas, a partir do surgimento da pandemia, tem enfrentado agravantes já conhecidos e identificados por órgãos públicos. Contudo, a dinâmica adotada ao combate do agravamento de consequências clínicas, epidemiológicas e sociais tem ido de encontro à uma realidade bem diferente aos aspectos pré-definidos por dados oficiais. Registros de fome, genocídios e contaminação em massa têm sido tópicos de discussões na mídia que, constantemente, questionam a população acerca da legitimidade do processo de garantia de direitos fundamentais quanto a viabilização de uma seguridade social equânime à população indigenista (Nicacio; Da Cruz Sousa et al., 2020).

Muitas são as situações que podem se mostrar nocivas ao contexto de aldeias e comunidades indígenas isoladas, dessa forma, este trabalho teve como objetivo realizar uma revisão integrativa de artigos científicos capaz de investigar a influência da pandemia no contexto da saúde nestas populações, a fim de identificar e categorizar possíveis agravantes capazes de explicar o real impacto da COVID-19 na construção de um bem-estar justo a este grupo populacional.

\section{Metodologia}

Este estudo trata-se de uma revisão integrativa qualitativa de caráter crítico acerca do impacto da pandemia do coronavírus na consolidação do bem-estar físico e social de populações indígenas do Brasil. Foram realizadas buscas nas bases de dados Scielo, BVS (Biblioteca Virtual em Saúde) e PubMed. Os descritores controlados utilizados no estudo constam no registro de Descritores em Ciências da Saúde (DeCS/MeSH) presentes na Biblioteca Virtual em Saúde (BVS), sendo estes os seguintes termos em inglês: "COVID-19" e "Brazil". Já o descritor não controlado (palavra-chave) utilizado à especificidade da busca nas bases de dados foi o termo "indigenous". Tais descritores foram ligados pelo operador booleano "AND", e os manuscritos foram selecionados através da leitura do resumo, sendo seu conteúdo, posteriormente, avaliado na íntegra. Foram incluídos todos os textos em língua inglesa e portuguesa publicados nos últimos 05 anos e que estão disponíveis para acesso gratuito. Foram excluídos os artigos duplicados, notas, pareceres, informativos, dissertações, trabalhos de conclusão de curso, 
resumos publicados em anais de congressos e artigos cujo conteúdo não explorou o impacto do COVID-19 na esfera social, cultural ou salutar de populações indígenas brasileiras. No total foram encontrados 22 artigos: 08 no Pubmed, 05 no Scielo e 09 na BVS. A seleção inicial dos artigos se deu por meio da leitura dos títulos e do resumo, onde, após constatada a adequação dos mesmos aos critérios desta pesquisa, restaram 10 artigos para a leitura na íntegra e composição da síntese objetivada por esta revisão (Figura 1).

Figura 1 - Fluxograma da síntese metodológica empregada.

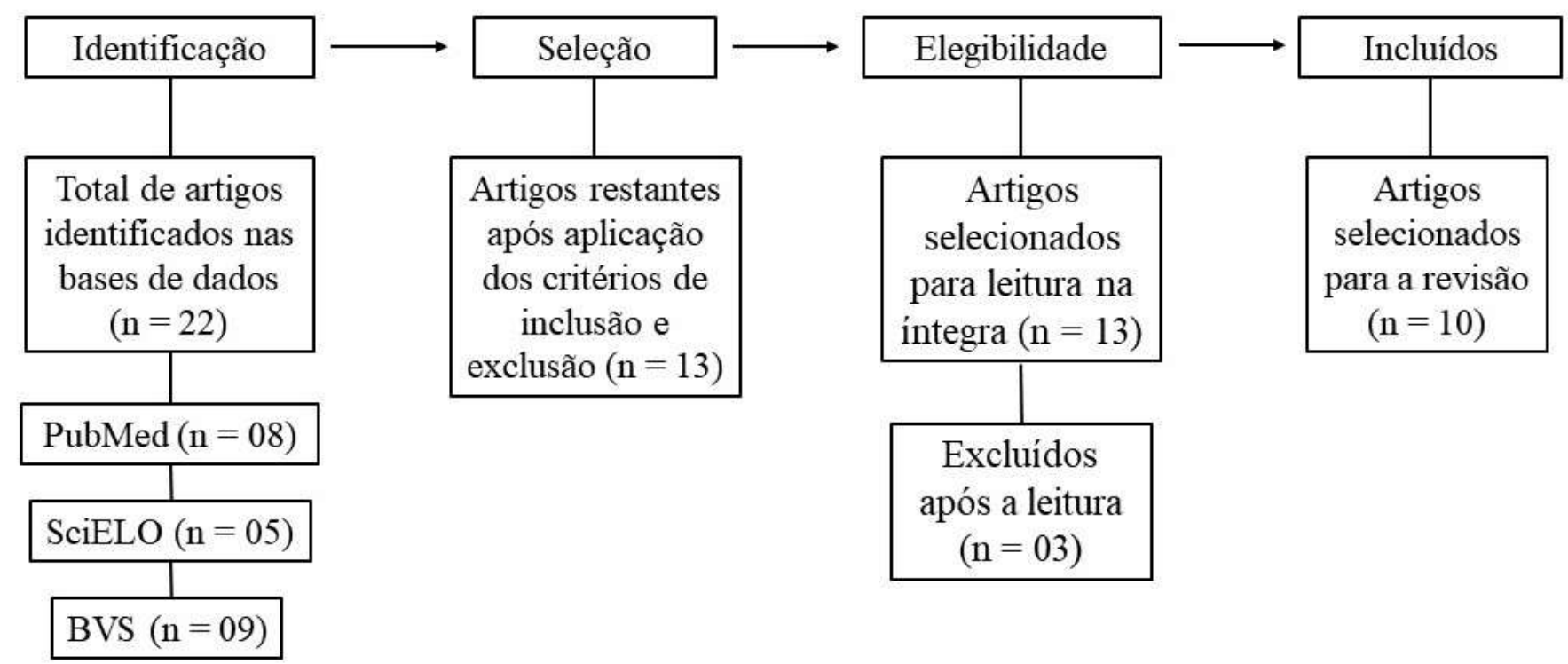

Fonte: Autores (2021).

\section{Resultados e Discussão}

Os principais achados desta investigação evidenciaram inúmeros fatores nocivos ao combate da COVID-19 na realidade indígena, onde a maioria deles refere-se à subnotificação de casos, às consequências obtidas a partir do êxodo de indígenas residentes de áreas urbanas até suas aldeias de origem, a falta de insumos alimentícios devido a entraves comerciais decorrentes do isolamento social, e ao medo constante da ameaça genocida ocasionada pela atuação de madeireiros na atividade extrativista predatória, que tem encontrado na vulnerabilidade dessas comunidades um cenário propício a tomada de territórios. O Quadro 1 expõe os principais achados desta revisão integrativa. 
Quadro 1 - Artigos considerados à revisão integrativa.

\begin{tabular}{|c|c|c|c|}
\hline Autor (a) & Título & Ano & Agravos evidenciados pela pandemia de Covid-19 \\
\hline Alves et al. & $\begin{array}{l}\text { Impact of COVID-19 on the indigenous } \\
\text { population of Brazil: A geo- } \\
\text { epidemiological study }\end{array}$ & 2021 & $\begin{array}{l}\text { Aldeias da região Norte e Centro-Oeste foram as } \\
\text { mais afetadas pela pandemia. }\end{array}$ \\
\hline Da Silva et al. & $\begin{array}{l}\text { Epidemiology of COVID-19 Among } \\
\text { Indigenous Populations in Brazil. }\end{array}$ & 2021 & $\begin{array}{l}\text { Priorização do uso de fitoterápicos no combate aos } \\
\text { sintomas do COVID-19. }\end{array}$ \\
\hline Da Silva et al. & $\begin{array}{l}\text { The Articulation of the Indigenous Peoples } \\
\text { of Brazil in Facing the Covid-19 Pandemic. }\end{array}$ & 2021 & $\begin{array}{l}\text { Apesar de inúmeras entraves, ações de prevenção e } \\
\text { promoção de saúde tem sido executadas em } \\
\text { territórios indígenas. }\end{array}$ \\
\hline Fellows et al. & $\begin{array}{l}\text { Under-reporting of COVID-19 cases among } \\
\text { Indigenous Peoples in Brazil: a new } \\
\text { expression of old inequalities. }\end{array}$ & 2021 & $\begin{array}{l}\text { Subnotificação de casos devido a negação étnica de } \\
\text { indígenas, e alocação dos mesmos em territórios } \\
\text { urbanos. }\end{array}$ \\
\hline Hillesheim et al. & $\begin{array}{c}\text { Síndrome respiratória aguda grave por } \\
\text { COVID-19 em crianças e adolescentes no } \\
\text { Brasil: perfil dos óbitos e letalidade } \\
\text { hospitalar até a } 38^{a} \text { Semana Epidemiológica } \\
\text { de } 2020\end{array}$ & 2020 & $\begin{array}{c}\text { Agravamentos em saúde devido as barreiras de } \\
\text { acesso, as condições sanitárias de aldeias, e a } \\
\text { migração indígena. }\end{array}$ \\
\hline Leite et al. & $\begin{array}{l}\text { Indigenous protagonism in the context of } \\
\text { food insecurity in times of Covid- } 19 \text {. }\end{array}$ & 2020 & $\begin{array}{c}\text { A redução da mobilidade entre aldeias e cidades } \\
\text { enfatizou a insegurança alimentar. }\end{array}$ \\
\hline Minalez et al. & $\begin{array}{l}\text { Fighting the invisible anaconda amidst a } \\
\text { war of conquest: notes of a genocide. }\end{array}$ & 2020 & $\begin{array}{l}\text { O deslocamento de aldeias favorece o extrativismo } \\
\text { predatório. }\end{array}$ \\
\hline Palamim et al. & $\begin{array}{l}\text { COVID-19 in the Indigenous Population of } \\
\text { Brazil. }\end{array}$ & 2020 & $\begin{array}{l}\text { Chefe de aldeias (caciques) estão entre o grupo mais } \\
\text { propício a complicações por COVID-19, devido a } \\
\text { idade avançada dos mesmos. }\end{array}$ \\
\hline Santos et al. & $\begin{array}{l}\text { A "total social fact": COVID-19 and } \\
\text { indigenous peoples in Brazil. }\end{array}$ & 2020 & $\begin{array}{l}\text { Aspectos socioculturais apresentam potencial risco de } \\
\text { contaminação em rituais e compartilhamento de } \\
\text { utensílios. }\end{array}$ \\
\hline Sardinha et al. & $\begin{array}{l}\text { Clinical characteristics of Severe Acute } \\
\text { Respiratory Syndrome by COVID-19 in } \\
\text { Indigenous of Brazil. }\end{array}$ & 2020 & $\begin{array}{l}\text { Principais sintomas observados nesta população: } \\
\text { febre, tosse, dor de garganta e dispneia. }\end{array}$ \\
\hline
\end{tabular}

Fonte: Autores (2021).

Dados notificados pelos Distritos Sanitários Especiais Indígenas (DSEI) apontaram que, entre março e outubro de 2020, as etnias que mais correram risco de contaminação e morte pela COVID-19 foram aquelas alocadas na região dos DSEIs Kaiapó do Pará, Altamira, Cuiabá, Kaiapó do Mato Grosso e Rio Tapajós; o que, em suma, abrange as regiões Norte e CentroOeste do país (Alves et al., 2021). Contudo, um fator agravante na identificação da realidade em populações indígenas, como descrito no estudo de Fellows et al. (2021), é a preocupante subnotificação de casos que podem ser justificados, dentre outros aspectos, pela negação da etnia indígena devido ao fato de muitos registros oficiais os classificarem como pardos. Atrelado a isso há o fato de existirem milhares de indígenas morando em áreas urbanas, o que impossibilita a precisão do registro de casos através da Secretaria Especial de Saúde Indígena (SESAI). Dados mais rigorosos acerca destes índices apontam desconformidade entre os números apresentados pela Coordenação das Organizações Indígenas da Amazônia Brasileira (casos confirmados $=25.356$; mortes $=670)$ em comparação com os dados do Ministério da Saúde $($ casos confirmados $=22.127$; mortes $=330$ ) que indicaram a ocorrência de subnotificação de 14 contaminações e de 103\% de mortes (Fellows et al., 2021).

No que diz respeito aos sinais e sintomas mais comuns observados em indígenas frente a Síndrome Respiratória Aguda Grave (ocasionada pela COVID-19), notou-se a recorrência de febre, tosse, dor de garganta e dispneia (sendo a dispneia e a saturação de $\mathrm{O}^{2}<95 \%$ mais frequente nos casos de morte), e afetando, principalmente, adultos e idosos do sexo masculino. Já as comorbidades mais prevalentes nestes grupos foram o diabetes mellitus e cardiopatias crônicas. Apesar destes dados serem muito semelhantes aos observados em populações não-indígenas, é necessário salientar que as políticas de saúde instituídas a este grupo não podem ser comparadas às estratégias adotadas em populações de grandes centros urbanos, uma vez 
que comunidades indígenas apresentam vulnerabilidades específicas capazes de potencializar o impacto da pandemia (Sardinha et al., 2020).

Hillesheim et al. (2020) atestou a alta taxa de letalidade entre crianças e adolescentes indígenas que foram internados com Síndrome Respiratória Aguda Grave (SRAG) pela COVID-19. Este agravante na população indígena pode estar relacionado às inúmeras barreiras de acesso aos serviços de saúde, assim como precárias condições sanitárias impostas aos mesmos, o que, constantemente, limitam a tomada de medidas de prevenção em saúde capazes de combater a disseminação do vírus. E tal letalidade, corriqueiramente, sofre influência de desigualdades na disponibilidade, distribuição e qualidade dos recursos destinados à saúde, o que exige a adoção de medidas adequadas às particularidades de inúmeros segmentos sociais.

Há estudos que indicam a pandemia como um acontecimento propício ao que se denomina "política de extermínio" favorecida pelas autoridades brasileiras frente ao agronegócio consolidado a qualquer custo, uma vez que o terror da propagação do coronavírus nos territórios indígenas tem favorecido o engrandecimento da elite rural a partir de projetos políticos favoráveis ao "genocídio, etnocídio e o ecocídio". Um exemplo disso tem sido o beneficiamento de economistas extrativistas, devido a migração de ocupantes de aldeias indígenas para regiões ainda mais isoladas, a fim de garantir o distanciamento social destes com o homem branco. Este deslocamento - viabilizado por grileiros - têm favorecido o acesso de economistas a terras vazias, antes povoadas, e que são alvos do extrativismo predatório há anos (Milanez, 2020).

A insegurança alimentar em aldeias também é um agravante ao quadro geral de comunidades vulneráveis, uma vez que isso pode ocasionar ou complicar doenças crônicas consideradas propícias à manifestação da COVID-19. O processo de isolamento social em aldeias - ainda que necessário - reduziu a mobilidade entre pequenas comunidades e as grandes metrópoles, o que afetou o abastecimento de determinados alimentos, acarretando em efeitos negativos na produção, aquisição e consumo de alimentos (Leite et al., 2020). Esta instabilidade só reforça a importância de se fortalecer a autonomia alimentar em aldeias, incentivando a agroecologia a fim de fortalecer bases locais e regionais de abastecimento, para assim minimizar a dependência exclusiva de ações solidárias que envolvam a doação de cestas básicas (Mondardo, 2020).

Ainda, a falta de tratamento adequado da água fornecida pelo saneamento básico brasileiro tem sido alvo de preocupações pela esfera estatal, uma vez que 53\% da população ainda não tem acesso à rede de esgoto, tornando-as propícias à sérias contaminações - principalmente em crianças residentes em regiões urbanizadas (Oliveira, 2015). Aliado a isto, existem fatos e costumes intrínsecos na dinâmica de convivência indígena que são considerados verdadeiros aliados à propagação do vírus entre estes indivíduos, a citar a coleta parcial de água com canecos ou vasilhames compartilhados, e a dificuldade na obtenção de insumos básicos de higiene pessoal, uma vez que as condições limitantes de transporte e compra destes produtos tem se deteriorado com a redução do funcionamento do mercado. O processo de migração indígena também pode ser considerado um fator de alto risco de contágio, uma vez que ao longo da pandemia muitos indígenas saíram da área urbana e voltaram às suas comunidades de origem (Hillesheim et al., 2020).

Sob a perspectiva de perda cultural frente ao risco de morte, é importante observar que boa parte das etnias são lideradas por um chefe (cacique) responsável por repassar, verbalmente, os ensinamentos hereditários de seus antepassados. E devido ao fato de os mesmos apresentarem uma idade avançada, estes estariam mais propícios a adquirirem quadros graves da COVID-19, tendo grande possibilidade de sucumbirem à doença (Palamim et al., 2020). Soma-se a isso aspectos socioculturais com potencial risco à contaminação interna nas aldeias como a grande quantidade de pessoas vivendo em um mesmo espaço, o compartilhamento de utensílios pessoais e a realização de cerimônias coletivas (Santos et al., 2020).

Outra característica observada em algumas etnias foi a resistência - principalmente entre os mais velhos - ao uso de medicamentos para o tratamento de sintomas. Muitos deles optaram pela administração da medicina tradicional como sendo o principal meio de amenizar as consequências do coronavírus: fumaça de colmeias em becos e cabanas para o extermínio de impurezas no ar, e o consumo de xaropes tradicionais para o fortalecimento do sistema imunológico são um destes exemplos. 
Por outro lado, há relatos que indicam que indivíduos mais jovens apresentam grande resistência a estas preparações naturais, aderindo ao uso de analgésicos sintéticos ou simplesmente não fazem uso de nenhuma substância - seja por conta do sabor desagradável destas misturas, ou devido ao distanciamento de suas raízes culturais (Da Silva et al., 2021).

Determinadas articulações políticas têm proporcionado uma regressão na construção da saúde indígena como um bem já consolidado. Um exemplo disso pode ser o Decreto 9.795 de 17 de maio de 2019, que trouxe modificações importantes no que se refere à atuação de departamentos específicos para a garantia de direitos salutares. A extinção do Departamento de Gestão da SESAI tem sido considerada uma tentativa do governo de incorporar o cuidado indígena ao contexto do cuidado à população em geral, esquecendo, entretanto, que o discurso de unificação do "cidadão universal brasileiro" desconsidera significativas iniquidades que os povos indígenas enfrentaram (e ainda enfrentam) em sua histórica trajetória pautada na busca pela equidade e efetivação de direitos básicos (Polidoro et al., 2020).

No entanto, em contraponto às disparidades citadas neste estudo, é necessário enfatizar que medidas de prevenção e promoção de saúde têm sido adotadas em aldeias, apesar da presença de características nocivas evidenciadas pela pandemia. Dentre as tomadas de ações que mais se destacam ao enfrentamento da COVID-19 entre indígenas, há o constante mapeamento da disseminação do vírus entre as comunidades, a identificação de possíveis vetores, e a busca pela garantia de acesso aos insumos de higiene pessoal. Outras medidas têm sido alvo de intensa sistematização e insistente incorporação no debate entre lideranças e agentes federais - tal como exemplificado neste texto -, a citar: a melhoria do saneamento básico, a constante realização de testes diagnósticos, a garantia de proteção territorial, a maior participação de lideranças indígenas na tomada de decisões, e, essencialmente, a efetivação de mudanças no processo de escuta e compreensão de crenças e costumes das populações autóctones (Da Silva et al., 2021).

\section{Considerações Finais}

Aspectos como barreiras geográficas, atuação de grileiros, subnotificação de casos, condições inadequadas de higiene e alimentação foram as principais variáveis encontradas nos estudos utilizados para esta revisão. Observou-se que a pandemia do coronavírus acentuou a presença de diversas desigualdades inerentes à história de sobrevivência dos povos indígenas, contudo, a incerteza de programas de vacinação, de acesso a serviços de saúde e a supressão da atuação de lideranças indígenas na formulação de políticas públicas, têm evidenciado uma instabilidade que antes era latente, mas que atualmente encontra-se exposta diante do período de crise econômico e salutar. $\mathrm{O}$ isolamento social como artifício facilitador à atuação de grileiros, a fome como agravamento de doenças crônicas, e o questionamento da visão holística da saúde indígena, também foram aspectos importantes abordados nos textos, o que enfatiza - ainda mais - a relevância em se olhar para as necessidades de aldeados a partir das singularidades impostas à solidificação de sua cidadania - tendo em vista a indissociação da territorialização, dos costumes e da cultura à realidade destes povos.

Há de se observar a interdependência de certas comunidades ao contato com não-indígenas no que se refere à garantia de assistência em saúde e até da prática econômica do ecoturismo - fatores inerentes ao desenvolvimento salutar e econômico destas populações -, e a partir disso proporcionar subsídios seguros para o combate das consequências remanescentes da pandemia. Ainda que os textos tenham indicado a tomada de medidas de prevenção e promoção em saúde por parte do governo a fim de minimizar os danos causados pela pandemia, é crucial a existência de um diálogo consistente entre as lideranças indígenas - que conhecem a realidade do seu povo a partir da vivência - com os Distritos Sanitários Especiais Indígenas (DSEI), em conformidade ao Ministério da Saúde/FUNASA, a fim de instituírem planejamentos viáveis de implementação a partir de uma ótica emergencial, dado a importância em se reduzir o número de novos casos e óbitos. Os dados levantados por este estudo também confluem ao descaso ideológico pregado pelo chefe de governo da República Federativa, cujo discurso enviesado por interesses políticos, minimiza a dimensão de problemas relevantes que afetam, principalmente, as minorias da 
sociedade brasileira.

\section{Referências}

Almeida, W. D. S. D., Szwarcwald, C. L., Malta, D. C., Barros, M. B. D. A., Souza, P. R. B. D., Azevedo, L. O., Romero, D., Lima, M. G., Damacena, G. N., Machado, I. E., Gomes, C. S., Pina, M. F., Gracie, R., Werneck, A. O. \& Silva, D. R. P. D. (2021). Mudanças nas condições socioeconômicas e de saúde dos brasileiros durante a pandemia de COVID-19. Revista Brasileira de Epidemiologia, 23(1), e200105. https://10.1590/1980-549720200105.

Alves, J. D., Abade, A. S., Peres, W. P., Borges, J. E., Santos, S. M., \& Scholze, A. R. (2021). Impact of COVID-19 on the indigenous population of Brazil: A geo-epidemiological study. medRxiv. https://10.1101/2021.01.12.21249703.

Belasco, A. G. S., \& Fonseca, C. D. D. (2020). Coronavirus. Revista brasileira de enfermagem, 73(2), e2020n2. https://10.1590/0034-7167-2020730201.

Cascella, M., Rajnik, M., Aleem, A., Dulebohn, S., \& Di Napoli, R. (2021). Features, evaluation, and treatment of coronavirus (COVID-19). StatPearls. https://www.statpearls.com/ArticleLibrary/viewarticle/52171.

Da Cruz Sousa, F., de Moraes, N. R., Quiqueto, A. M., \& Teodoro, V. B. (2020). COVID-19 E POVOS INDÍGENAS: Aspectos de seguridade social. Revista Observatório, 6(2), a12pt-a12pt. https://doi.org/10.20873/uft.2447-4266.2020v6n2a12pt.

Da Silva, L. L., Nascimento, P. E., Araújo, O. C. G., \& Pereira, T. M. G. (2021). The Articulation of the Indigenous Peoples of Brazil in Facing the Covid-19 Pandemic. Frontiers in Sociology, 6(1), 611336. https://doi.org/10.3389/fsoc.2021.611336.

Da Silva, M. G., Pereira, P. M. B., Portela, W. F., Daros, G. C., de Almeida Barbosa, C. R., Vanassi, B. M., Parma, G. O. C., Bitencourt, R. M. \& Iser, B. P. M. (2021). Epidemiology of COVID-19 Among Indigenous Populations in Brazil. Journal of Racial and Ethnic Health Disparities, 1-7. https://doi.org/10.1007/s40615-021-01035-2.

Fellows, M., Paye, V., Alencar, A., Nicácio, M., Castro, I., Coelho, M. E., Silva, C. V. J., Bandeira, M., Lourival, R. \& Basta, P. C. (2021). Under-reporting of COVID-19 cases among Indigenous Peoples in Brazil: a new expression of old inequalities. Frontiers in Psychiatry, 12(1), 352. https://doi.org/10.3389/fpsyt.2021.638359.

Hillesheim, D., Tomasi, Y. T., Figueiró, T. H., \& Paiva, K. M. D. (2020). Síndrome respiratória aguda grave por COVID-19 em crianças e adolescentes no Brasil: perfil dos óbitos e letalidade hospitalar até a 38 Semana Epidemiológica de 2020. Epidemiologia e Serviços de Saúde, 29(1), e2020644. https://doi.org/10.1590/S1679-49742020000500021.

Leite, M. S., Ferreira, A. A., Bresan, D., Araujo, J. R., Tavares, I. D. N., \& Santos, R. V. (2020). Indigenous protagonism in the context of food insecurity in times of Covid-19. Revista de Nutrição, 33. https://doi.org/10.1590/1678-9865202033e200171.

Marinho, G., Pontes, A. L. de M. (2017). Políticas de saúde indígena no Brasil: do modelo assistencial à representação política. Langdon EJ, Cardoso MD, organizadores, Saúde indígena: políticas comparadas na América Latina. Florianópolis: Ed. da UFSC, 83-106. https://10.1590/0102-311X00024117.

Mendes, A. M., Leite, M. S., Langdon, E. J., \& Grisotti, M. (2018). O desafio da atenção primária na saúde indígena no Brasil. Revista Panamericana de Salud Pública, 42(1), e184. https://doi.org/10.26633/RPSP.2018.184.

Milanez, F. (2020). Fighting the invisible anaconda amidst a war of conquest: notes of a genocide. Ambiente \& Sociedade, 23. http://dx.doi.org/10.1590/18094422asoc20200116vu2020L3ID.

Mondardo, M. (2020). Povos indígenas e comunidades tradicionais em tempos de pandemia da Covid-19 no Brasil: estratégias de luta e rexistência. Finisterra, 55(115), 81-88. https://doi.org/10.18055/Finis20364.

Nicacio, M. (2020). Descaso com a Covid-19 pode exterminar povos indígenas. Folha de São Paulo, 15 abr. 2020.2 Opinião. https://www1.folha.uol.com.br/opiniao/2020/04/descaso-com-a-covid-19-pode-exterminar-povos-indigenas.shtml.

Oliveira, J. P. M., Oliveira, J. M., Barreto, E. D. S., Silva, S. S. D., Silva, S. S., \& Maracajá, P. B. (2015). Saúde/doença: as consequências da falta de saneamento básico. Informativo Técnico do Semiárido, 9(2), 23-29. https://www.gvaa.com.br/revista/index.php/INTESA/article/view/3592/3239.

Palamim, C. V. C., Ortega, M. M., \& Marson, F. A. L. (2020). COVID-19 in the Indigenous Population of Brazil. Journal of racial and ethnic health disparities, 7(6), 1053-1058. https://doi.org/10.1007/s40615-020-00885-6.

Polidoro, M., de Assis Mendonça, F., Meneghel, S. N., Alves-Brito, A., Gonçalves, M., Bairros, F., \& Canavese, D. (2020). Territories under siege: risks of the decimation of indigenous and Quilombolas peoples in the context of COVID-19 in South Brazil. Journal of Racial and Ethnic Health Disparities, 1-11. https://doi.org/10.1007/s40615-020-00868-7.

Santos, R. V., Pontes, A. L., \& Coimbra, C. E. (2020). A "total social fact": COVID-19 and indigenous peoples in Brazil. Cadernos de Saúde Pública, 36(10), e00268220. https://doi.org/10.1590/0102-311X00268220.

Sardinha, D. M., Karla, V. B., Ferreira, A. L., Garcez, J. C., Ueno, T. M., Rodrigues, Y. C., Santos, A. L. S., Loiola, R. S. P., Guimarães, R. J. P. S. \& Lima, L. N. (2021). Clinical and spatial characteristics of Severe Acute Respiratory Syndrome by COVID-19 in Indigenous of Brazil. medRxiv, 2020-10. https://doi.org/10.1101/2020.10.24.20218701.

Scalco, N., Nunes, J. A., \& Louvison, M. (2020). Controle social no Subsistema de Atenção à Saúde Indígena: uma estrutura silenciada. Saúde e Sociedade, 29, e200400. https://doi.org/10.1590/S0104-12902020200400. 